\title{
Granulomatous Rosacea
}

\author{
Jose Aneiros-Fernandez ${ }^{\mathrm{a}, \mathrm{d}}$, Salvador Arias-Santiago ${ }^{\mathrm{b}}$, Barbara Cancela-Diez ${ }^{\mathrm{c}}$, \\ Francisco O'Valle ${ }^{a}$, Jose Aneiros-Cachaza ${ }^{a}$
}

\begin{abstract}
Granulomatous rosacea is considered as a variant of rosacea with unknown etiology. For diagnosis, skin biopsy is needed. We present a male patient of 54 years old who presented erythematous papular lesions which have become nodular lesions.
\end{abstract}

Keywords: Granulomatous Rosacea; Treatment; Differential diagnosis; Seborreic dermatitis; Sarcoidosis; Cutaneous leishmaniasis; Granulomatous periorificial dermatitis

\section{Introduction}

Rosacea is a chronic inflammatory infiltrate of unknown etiology, characterized clinically by presenting transient or persistent facial erythema, telangiectasia, papules and pustules. Clinically it is classified as telangiectatic erythematous, papulopustular and granulomatous $[1,2]$.

We present a male patient of 54 years old who presented erythematous papular lesions which have become nodular lesions.

\section{Case Report}

An otherwise healthy 54-year-old man presented with a

Manuscript accepted for publication November 18, 2010

${ }^{a}$ Department of Pathology, University Hospital, Granada, Spain

${ }^{b}$ Department of Dermatology, University Hospital, Granada, Spain

${ }^{\mathrm{c}}$ Department of Pharmacology, University Hospital, Granada, Spain

${ }^{\mathrm{d} C}$ Corresponding author: Department of Pathology, University Hospital,

Avd. Madrid S/N, CP: 18012, Granada, Spain.

Email: janeirosf@hotmail.com

doi:10.4021/jmc98e month history of itching lesions on the face. Physical examination revealed erythematous papules and plaques with scales on the cheek, forehead and eyelids with blepharitis (Fig. 1). The remainder of the skin exam was unremarkable. Laboratory testing revealed an increase of cholesterol level. ANA antibodies were negative. Histologic section showed hyperkeratotic stratum corneum with focal parakeratosis. The epidermis showed an irregular acanthosis. The superficial and middle dermis showed a granulomatous inflammatory infiltrate which was perivascular and predominantly periadnexal. The granulomatous component presented some multinucleated giant cells. Some lymphocytes were impaired adnexal dermoepidermal interface (Fig. 2, 3).

\section{Discussion}

Granulomatous rosacea is a rare caseating granulomatous variant of rosacea that may present similar to other granulomatous disease. The etiology of rosacea is unknown. However, several factors, such as vasculature, climatic exposures, dermal matrix degeneration, chemicals and ingested agents, pilosebaceous unit abnormalities, microbial organisms, ferritin expression, reactive oxygen species, and dysfunction of antimicrobial peptides, likely play a role in its development [3]. Furthermore, the distinct subtype of rosacea is likely de-

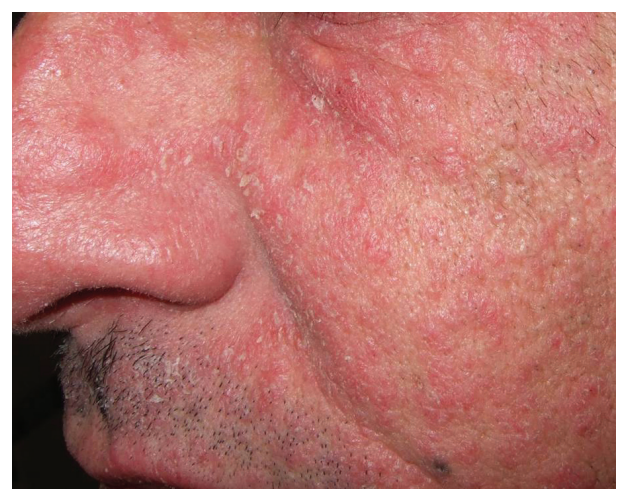

Figure 1. Erythematous papules and plaques with scales on the cheek, forehead and eyelids with blepharitis. 


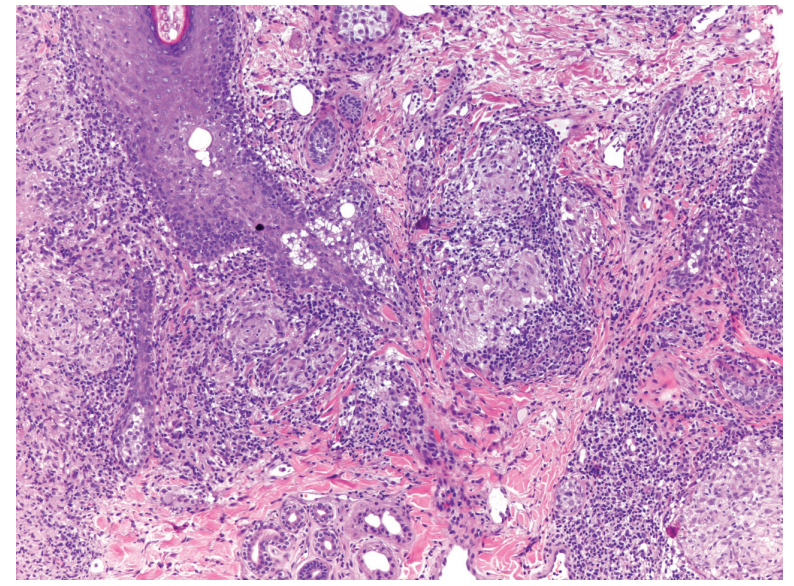

Figure 2. Histological sections showed epithelioid histiocytes and multinucleate giant cells in granulomatous and perifollicular lymphohistiocytic infiltrate (H and E, x 200).

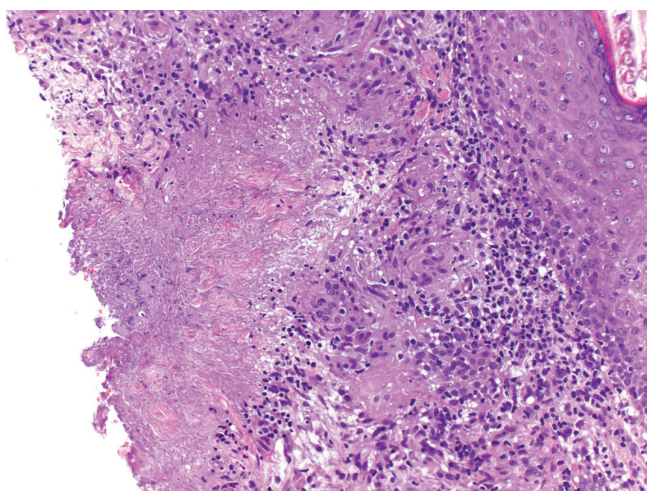

Figure 3. Higher magnification revealed tuberculoid granulomas with caseous necrosis ( $\mathrm{H}$ and $\mathrm{E}, \mathrm{x} 400)$.

termined by a patient's unique sensitivity to these triggers.

Clinically, granulomatous rosacea appears to be a distinctive papular form of rosacea that is found primarily on the butterfly and perioral areas. These discrete papules may appear as yellowish-brown hard nodules on diascopy, and may be accompanied by marked erythema. The size of the lesions may vary, and may be present at other areas of the body besides the above mentioned [4]. Histological examination presents epithelioid histiocytes and multinucleate giant cells in tuberculoid granulomatous, which may be centered on ruptured hair follicles. Non-pustular lesions show a nonspecific perivascular and perifollicular lymphohistiocytic infiltrate accompanied by occasional multinucleated cells, plasma cells, neutrophils, and eosinophils. Papulopustular lesions show more pronounced granulomatous inflammation and occasional perifollicular abscesses [1]. Some of the additional tests that pathologists may utilize include special stains such as an Acid Fast, Fite, Gram, Warthin-Starry, PAS, and GMS stains. In addition, polarization with refractile light examination may be helpful in identifying some causes like a foreign body with giant cell reaction.

The differential diagnosis with granulomatous periorificial dermatitis is an eruption characterized by grouped papules, pustules, and diffuse erythema in prepubertal children [3]. Seborreic dermatitis is a papulosquamous disorder characterized by greasy scaling over inflamed skin on scalp, face and trunk. Activity is increased in winter and spring with remissions commonly occurring in summer. Sarcoidosis is inflammatory systemic disorder with typically pulmonary involvement but can affect almost any organ. A biopsy showed well-circumscribed nodular collections of epithelioid histiocytes in the dermis without peripheral lymphocytic infiltrate (naked granulomas). Cutaneous leishmaniasis showed epidermis hyperplasia with dense granulomatous infiltrate composed of histiocytes, neutrophils, lymphocytes and plasma cells. Focal pale areas with organism-laden macrophages showed amastigote form of leishmania in cytoplasm of macrophages, especially in the subepidermal component of infiltrate in the middle of lesion.

Treatment options include isotretinoin, a drug which elicits a response with several subtypes of rosacea including severe granulomatous rosacea. The treatment leads to the reduction of inflammatory lesions, erythema and telangiectasia. Doxycycline (100 - $200 \mathrm{mg}$ per day) is an antibiotic that has been used for rosacea for decades. Also it has other non-antibiotic properties such as inhibition of angiogenesis, neutrophil chemotaxis, pro-inflammatory cytokines, and metalloproteinases that contribute to the clinical improvement. Topical metronidazole is considered the first choice for topical therapy of mild rosacea. Azelaic acid is effective and safety and has been demonstrated for the treatment of moderate to severe rosacea applied as $15 \%$ or $20 \%$ cream [5].

In the present case, treatment with isotretinoin $20 \mathrm{mg}$ per day was prescribed with good response.

\section{Conflict of Interest}

The authors have no conflict of interest to declare.

\section{Acknowledgement}

All authors have participated sufficiently to take public responsibility for appropriate portions of the work.

\section{References}

1. Crawford GH, Pelle MT, James WD. Rosacea: I. Etiology, pathogenesis, and subtype classification. J Am Acad 
Dermatol 2004;51(3):327-341; quiz 342-324.

2. Wilkin J, Dahl M, Detmar M, Drake L, Liang MH, Odom R, Powell F. Standard grading system for rosacea: report of the National Rosacea Society Expert Committee on the classification and staging of rosacea. J Am Acad Dermatol 2004;50(6):907-912.

3. Khokhar O, Khachemoune A. A case of granulomatous rosacea: sorting granulomatous rosacea from other granulomatous diseases that affect the face. Dermatol Online J 2004;10(1):6.

4. Sanchez JL, Berlingeri-Ramos AC, Dueno DV. Granulomatous rosacea. Am J Dermatopathol 2008;30(1):6-9.

5. Malik R, Quirk CJ. Topical applications and perioral dermatitis. Australas J Dermatol 2000;41(1):34-38. 